\title{
ROSETTA's Prospects for Discovering Evidence of Cometary Biology
}

\section{Chandra Wickramasinghe*}

Buckingham Centre for Astrobiology, University of Buckingham, UK

\begin{abstract}
The long-awaited rendezvous of ROSETTA with its target Comet 67P/Churyumov-Gerasimenko is expected at best to give indirect evidence for cometary panspermia and the presence of microorganisms in comets.
\end{abstract}

Keywords: Comets; Panspermia; ROSETTA mission

\section{Introduction}

ROSETTA's decade-long mission to Comet 67P/ChuryumovGerasimenko following its launch in 2004 is fast approaching its climax. In January this year (2014) the spacecraft exited a phase of hibernation and began to gear up for its ultimate landing (the lander Philae) on the comet's surface. The imaging of the nucleus accomplished to date has revealed a smallish sized comet of average diameter $4 \mathrm{~km}$ rotating with a period of 12.7 hours. Further results of the mission as they emerge will watched with eager anticipation, in particular for signs of any connections that might relate to our own origins.

Since the first space exploration of a comet - Comet Halley - in 1986 - an earlier entrenched paradigm of a 'dirty ice comet' has been gradually replaced by a class of model that is now widely admitted to have a possible link to an origin of life on the Earth. The evidence of liferelated organic material in Comet Halley was dramatically displayed both in ground-based infrared spectra, and in mass spectroscopy carried by the Giotto spacecraft $[1,2]$. The comet's dark non-reflecting surface was probably composed of a tarry residue overlying a frozen water-ice dominated terrain. The estimated visual albedo of $\mathrm{A}=0.1$ is consistent with $10 \%$ of the surface disrupted so as to expose underlying ice with $A=1$. Similar data relating to the dominance of complex organic molecules in comets have been confirmed since 1986. Both the Deep Impact Mission of 2005, involving an impact or crashing onto Comet Tempel 1, and the Stardust Mission, which led to a sample return in 2006, confirmed that comets do indeed carry material that is relevant to biology [2]. The contentious issue relates to the presence of fullyfledged microbial life that seeded the Earth and possibly other planets in the galaxy.

Recent detections of exoplanets by the Kepler Mission [3], combined with independent microlensing data [4] have led to an estimated total population of planetary systems of some 140 billion in the galaxy. The close separation of neighboring habitable planetary systems permits the efficient exchange of comets and bolides by well-attested dynamical processes [5], thus greatly strengthens the case for Cometary Panspermia. The recent discovery of cometary bolides (L-chondrites) in terrestrial sediments dated at 470 million years ago, coinciding with the time of the most extensive explosion of species in the geological record, gives further credence to a comet-life connection [6].

The idea of comets carrying bacteria and viruses is, however, still viewed with scepticism in orthodox circles; for this reason no explicit life detection experiment was included in the ROSETTA mission package. Although the author and Max Wallis were included in the original ROSETTA Radio Science Team, the lack of any interest in biological detection did not encourage us to continue our participation. The best that could be accomplished with the on-board ROSETTA equipment would be to secure indirect evidence that might point to biology.
The recent report [7] that Comet Churyumov-Gerasimenko was losing water prodigiously at a rate of $300 \mathrm{~g} / \mathrm{s}$ could be interpreted as such evidence, implying the resumption of subsurface microbiology as the comet approaches perihelion. On 6 June 2014 the comet was at a distance of $\mathrm{R}=5.83 \times 10^{13} \mathrm{~cm}$ from the sun. The comet's rapid rotation with a period of 12.7 hours implies that it intercepts solar energy on one hemisphere whist re-emitting infrared radiation over the entire surface. Thus we have

$$
(1-A) \frac{L}{4 \pi R^{2}}\left(\pi \mathrm{r}^{2}\right)=\Theta \sigma T^{4}\left(4 \pi \mathrm{r}^{2}\right)
$$

where $A$ is the optical albedo, $\Theta$ is the infrared emissivity, $L$ is the solar luminosity, $T$ is the temperature, $r$ is the comet's radius (assumed spherical) and $\sigma$ is the Stefan-Boltzman constant. The equation (1) gives the result $T=130 \mathrm{~K}$, assuming $A=0.1$ (as in Halley's comet) and $\Theta=1$. The rate of loss of $\mathrm{H}_{2} \mathrm{O}$ from a water-ice surface at temperature $T$ can be readily shown to be

$$
\left(\frac{m_{H 2 o}}{k T}\right)^{\frac{1}{2}} \rho(T) .4 \Pi \mathrm{r}^{2} f g s^{-1}
$$

where $p(T)$ is the saturation vapour pressure of water-ice and $f$ is the fraction of the cometary surface comprised of exposed water-ice. With appropriate values of the physical constants this becomes

$$
8.56 \times 10^{8} f \rho(T) g s^{-1}
$$

For a surface crusted over with a carbonaceous skin, as in the case of Comet Halley or Comet Temple 2, possessing an albedo of 0.1 it would be reasonable to set $\mathrm{f}=0.1$, which case we have an $\mathrm{H}_{2} \mathrm{O}$ outgassing rate of

$$
8.56 \times 10^{7} \rho(T) g s^{-1}
$$

With the saturation vapour pressure of water at $130 \mathrm{~K}$ being $10^{-7}$ dyne $\mathrm{cm}^{-2}$ (from tabulations and calculations by the author) the water emission rate is estimated at less than $10 \mathrm{~g} \mathrm{~cm}^{-3}, 30$ times less than has actually been found [7].

In spite of the uncertainties inherent in the present calculation, it

*Corresponding author: Chandra Wickramasinghe, Buckingham Centre for Astrobiology, University of Buckingham, UK, Tel: 44-777-838-9243; E-mail: ncwick@gmail.com

Received July 16, 2014; Accepted July 22, 2014; Published July 24, 2014

Citation: Wickramasinghe C (2014) ROSETTA's Prospects for Discovering Evidence of Cometary Biology. Astrobiol Outreach 2: 118. doi: 10.4172/23322519.1000118

Copyright: (c) 2014 Wickramasinghe C. This is an open-access article distributed under the terms of the Creative Commons Attribution License, which permits unrestricted use, distribution, and reproduction in any medium, provided the original author and source are credited. 
Citation: Wickramasinghe C (2014) ROSETTA's Prospects for Discovering Evidence of Cometary Biology. Astrobiol Outreach 2: 118. doi: 10.4172/2332-2519.1000118

Page 2 of 2

is possible to interpret the surprisingly large outflow of water observed from the Comet Churyumov-Gerasimenko on June 6, 2014, when the comet was between Jupiter and Mars, as being indicative of biology. Although microorganisms in comets are likely to be in a frozen dormant state at aphelion, a sporadic resumption of metabolism will occur near perihelion if subsurface melting can take place. Metabolism builds up subsurface gas pressures of thousands of atmospheres, which is enough to cause cracks in the surface crust releasing gas and dust. Such melting could occur due to increased solar radiation or, on occasion when impacts of smaller bodies transfer kinetic energy that can be converted to heat. Comet Hale-Bopp showed sporadic activity when it was outside the orbit of Jupiter. We can argue that this activity in Comet Hale-Bopp, as well as the new evidence from Comet Churyumov-Gerasimenko, point to the resumption of bacterial activity [8].

As we await the descent of the Philae lander onto Comet Churyumov-Gerasimenko with bated breath, there would inevitably be many surprises in store. The recent discovery that the comet has a "double" structure and is probably a contact binary may be the first of many to come. It is to be hoped that more indirect evidence of biology will be forthcoming as the ROSETTA mission draws to a conclusion this year.

\section{References}

1. Wickramasinghe DT and Allen DA (1986) Detection of organic grains in comet Halley. Nature 323: 44-46.

2. Wickramasinghe JT, Wickramasinghe NC, Napier WM (2011) Comets and the Origins of Life. World Scientific Publ., Singapore.

3. Kopparapu R (2013) A revised estimate of the occurrence rate of terrestrial planets in the habitable zones around kepler m-dwarfs.

4. MOA Group (2011) The microlensing observations in astrophysics. Nature 473 349-352

5. Edward B, Amaya MM, Renu M, Dmitry S (2012) Chaotic exchange of solid material between planetary systems: implications for lithopanspermia. Astrobiology 12: 754-774.

6. Birger S, Gary RH, Matthias MMM, Bernhard PE, Ross PC. et al. (2014) A fossil Winonaite-like meteorite in Ordovician limestone: a piece of the impactor that broke up the L-chondrite parent body. Earth \& Planet. Sci. Lett 400: 145-152.

7. http://sci.esa.int/rosetta/54251-first-detection-of-water-vapour/

8. Wickramasinghe NC, Hoyle F, Lloyd D (1996) Eruptions of Comet Hale-Bopp at 6.5 AU. Astrophys. Space Sci. 240: 161-165. 